\title{
Synthesis, Spectral Characterization and Antimicrobial Studies of New Hybrid Heterocyclic Compounds Bearing 1H-benzimidazol-2-yl Thiomethyl Motif
}

\author{
S. R. DESHPANDE*, P. S. CHAVAN, S. N. NAGARALE AND M. V. PATIL \\ Division of Medicinal and Pharmaceutical Chemistry, HSK College of Pharmacy, BVVS Old Campus, Bagalkot-587 101, India
}

Deshpande, et al:: Compounds with 1H-benzimidazol-2-yl Thiomethyl Motif

\begin{abstract}
To understand the biological importance of heterocyclic cores, novel 1 H-benzimidazol-2-yl thiomethyl incorporated hybrid compounds, 2-(benzimidazol-2-ylthiomethyl)-5-aryl-1,3,4-oxadizoles and 1-(2-(1H-benzo[d]imidazol-2-ylthio)acetyl)pyridazine/phthalazinediones were designed using molecular hybridization technique, synthesized and characterized. The compounds were screened for in vitro antimicrobial activity using the serial dilution technique and were found to exhibit weak antitubercular activity, excellent to moderate antibacterial and better antifungal activities against some tested organisms in comparison to the standard drugs. Thus, some of the title compounds demonstrated antimicrobial activity.
\end{abstract}

Key words: 1,3,4-oxadiazole, phthalazine, pyridazine, molecular hybridization, serial dilution, antitubercular drugs

Many infections are caused by pathogenic organisms like bacteria, fungi, and viruses. Even though antibiotics prescribed for the treatment of such diseases are lifesaving, some of these have harmful side effects leading to allergy, anaphylactic reaction, superinfections, destruction of normal bacterial flora of the body and selective toxicities like anaemia and renal damage. Moreover, the development of multiple drug resistance by causative organisms with time makes the existing antibiotics ineffective ${ }^{[1]}$. Therefore, development of effective, safe and economic antimicrobial agents is the need of the hour.

Heterocycles, over the years, have contributed to mankind to understand life processes and improving the quality of life. Exploration of new heterocycles that can interact with multiple biological targets remains an intriguing scientific endeavour. In particular, sulphur and nitrogen containing heterocycles remained to be the mainstay of continued interest by researchers ${ }^{[2]}$. Being structural surrogates of nucleotides, benzimidazole derivatives exhibited the potential to interact with proteins, enzymes and receptors. Substituted benzimidazoles were found to possess various biological activities such as antibacterial ${ }^{[3]}$, antiinflammatory ${ }^{[4]}$, antifungal ${ }^{[5]}$ antitubercular ${ }^{[6]}$, anticancer ${ }^{[7]}$, anticonvulsant and antidiabetic ${ }^{[8]}$. A number of 2-thio-
1H-benzimidazole derivatives have been demonstrated to exhibit potent antiparasitic ${ }^{[9,10]}$ activities. Diversely 2,5-disubstituted-1,3,4-oxadiazoles have not only been reported to possess antibacterial[11,12], antifunga ${ }^{[13]}$ and antibrucella ${ }^{[14]}$ actions but also served as building blocks for heterocyclic molecules. Likewise, compounds consisting of pyridazine and phthalazine cores too were reported to exhibit antiplatelet ${ }^{[15]}$, antitumor ${ }^{[16]}$ and antifungal ${ }^{[17]}$ activities.

Moreover, it is noticed in many instances that incorporation of a biologically active moiety into another active molecule could result in altered biological activity due to changes brought in the physicochemical properties by the incorporated moiety ${ }^{[18-20]}$. Molecular hybridization is an emerging strategy in drug design and development that involves combination of distinct pharmacophore moieties of different active molecules to produce a new hybrid compound with improved affinity and efficacy, when compared to

This is an open access article distributed under the terms of the Creative Commons Attribution-NonCommercial-ShareAlike 3.0 License, which allows others to remix, tweak, and build upon the work non-commercially, as long as the author is credited and the new creations are licensed under the identical terms

Accepted 15 April 2017

Revised 03 January 2017

Received 11 August 2016

Indian J Pharm Sci 2017;79(3):385-394 
the parent molecules. It is useful in the design of new optimized ligands and prototypes with new molecular architectures. This strategy can result in compounds with modified selectivity profile, different and/or dual modes of action and reduced side effects ${ }^{[21-23]}$. Enthused by these facts, the present study has been taken up to synthesize an effective and possibly safer hybrid molecular framework consisting two active scaffolds, 1,3,4-oxadiazole/pyridazine/phthalazine and 1H-benzimidazol-2-ylthiomethyl motif, based on molecular hybridization strategy followed by structural characterization and antimicrobial evaluation.

\section{MATERIALS AND METHODS}

The 1H-2-mercaptobenzimidazole and other chemicals purchased from S. D. Fine Chem., Mumbai, India were of laboratory reagent grade and used as received. Brain heart infusion (BHI) and Middlebrook 7H9 broths were obtained from HiMedia Laboratories Pvt. Ltd., Mumbai, India. Alamar Blue was purchased from Sigma-Aldrich, St. Louis, USA. Ciprofloxacin and fluconazole were obtained as gift samples from Lanova Chem., Mumbai, India while isoniazid (INH) was from KP Pharmaceuticals, Bidar, India.

Progress of the reaction and purity of the products were ascertained by thin-layer chromatography (TLC) using silica gel $G$ as stationary phase and various solvent combinations as mobile phase; the spots were visualized by iodine vapours. All yields refer to crude products before purification. Melting points were determined in an open capillary melting point apparatus, Veego VMP-DS, Mumbai, India and are uncorrected. The infrared (IR) spectra were taken on Fourier transform infrared spectroscopy (FTIR) 8400S (Shimadzu Corporation, Tokyo, Japan) using the $\mathrm{KBr}$ pellet technique. Proton nuclear magnetic resonance $\left({ }^{1} \mathrm{H}\right.$ NMR) spectra were obtained in DMSO-d6 on Bruker AvanceII $400 \mathrm{MHz}$ spectrometer (Illinois, USA). Chemical shifts were measured on $\delta$ scale in parts per million (ppm) downfield to tetramethylsilane. Peak multiplicities were indicated as $\mathrm{s}$ (singlet), $\mathrm{d}$ (doublet), dd (doublet of doublet), $\mathrm{t}$ (triplet) and $\mathrm{m}$ (multiplet). Carbon-13 nuclear magnetic resonance $\left({ }^{13} \mathrm{CNMR}\right)$ spectra were taken at $50 \mathrm{MHz}$. The mass spectra were recorded on AB Sciex API 2000 LC/ MS (Framingham, USA) by ESI technique. CLogP values were computed from ChemDraw software ${ }^{[24]}$. Staphylococcus aureus ATCC 25923, Enterococcus faecalis ATCC 29212, Escherichia coli ATCC 25922, Klebsiella pneumoniae ATCC 700603, Aspergillus niger ATCC 1015, Candida albicans ATCC 10231 and Mycobacterium tuberculosis H37Rv ATCC 25618 were used for antimicrobial studies.

\section{Preparation of 2-(1H-benzo[d]imidazol-2-ylthio) acetic acid 2:}

A mixture of 2-mercapto benzimidazole ( $15 \mathrm{~g}, 0.1 \mathrm{~mol})$, sodium hydroxide (4 g, $0.1 \mathrm{~mol})$ and alcohol $(95 \%$, $150 \mathrm{ml}$ ) was refluxed for $1 \mathrm{~h}$ and then cooled to room temperature. Chloroacetic acid $(9.14 \mathrm{~g}, 0.1 \mathrm{~mol})$ was added to reaction mixture and refluxed again for $4-5 \mathrm{~h}$, cooled and poured into ice cold water, acidified with dilute acetic acid and kept in refrigerator overnight. The solid separated as glistening white crystals was filtered, washed with cold water and recrystallized by ethanol. Yield: 93\%; melting point: 203-205.

\section{Preparation of 2-(1H-benzo[d]imidazol-2-ylthio) acetyl chloride 3:}

To compound 2 ( $20 \mathrm{~g}, 0.1 \mathrm{~mol})$ thionyl chloride $(21.6$ $\mathrm{ml}, 0.12 \mathrm{~mol}$ ) was added slowly during $1 \mathrm{~h}$ under reflux. The contents were mixed well during this period. The excess thionyl chloride was distilled off and the product was cooled and dried. Yield: $71 \%$; melting point: $300^{\circ}$.

\section{Preparation of 2-(1H-benzo[d]imidazol-2-ylthio) aceto hydrazide 4:}

A mixture of compound 3 (19 g, $0.88 \mathrm{~mol})$ and hydrazine hydrate (66 g, $1.32 \mathrm{~mol}$ ) was refluxed for 15 min. Ethanol (95\%) was added to get clear solution and refluxed further for $3 \mathrm{~h}$. The excess of ethanol was distilled off and cooled. The obtained solid was recrystallized from ethanol (95\%). Yield: 63\%; melting point: $159-162^{\circ}$.

\section{Preparation of 1,3,4-oxadiazole derivatives 5a-5j:}

A mixture of compound 4 (2.67 g, $0.012 \mathrm{~mol})$, aromatic acid $(0.01 \mathrm{~mol})$ and phosphorus oxy chloride $(2 \mathrm{ml}$, $0.01 \mathrm{~mol}$ ) was refluxed for $5 \mathrm{~h}$ and cooled. The reaction mixture was poured into ice water mixture, neutralized with dilute $\mathrm{NaHCO}_{3}$. The solid obtained was filtered, washed with cold water, dried and recrystallized from ethanol (95\%).

2-((1 H-benzo[d]imidazol-2-ylthio)methyl)-5phenyl-1,3,4-oxadiazole) 5a:

Prepared from benzoic acid $(1.22 \mathrm{~g}, 0.01 \mathrm{~mol})$ as brown needles; yield: $32 \%$; IR, $\mathrm{cm}^{-1}: 3173.64(\mathrm{NH}$ str), 3085.45 (ArCH str), $1675.16(\mathrm{C}=\mathrm{N}$ str), 1382.18 (NH bend), 744.57 (CS str); ${ }^{1} \mathrm{H}$ NMR, $\delta 11.52$ (s, 1H, 1'H), 8.26 (s, 2H, 2', 6'H), 8.11-8.09 (m, 5H, 5', 8', 
3", 4", 5'H), 7.69-7.67 (d, 2H, J=8.2 Hz, 6', 7'H), 3.98 $\left(\mathrm{s}, 2 \mathrm{H}, \mathrm{SCH}_{2}\right) ;{ }^{13} \mathrm{C} \mathrm{NMR}, \delta 163.8$ (C5), 158.2 (C2), 148.3 (C2'), 138.2 (C4', 9'), 129.6 (C3", 5”), 128.1 (C2", 4", 6"), 122.6 (C1', 6', 7'), 114.8 (C5', 8'), 35.2 (CS); MS, m/z: $308.09\left(100 \%, \mathrm{M}^{+}\right), 309.07$ (18.2\%, $\left.\mathrm{M}^{+}+1\right), 310.06\left(3.9 \%, \mathrm{M}^{+}+2\right),(\mathrm{M})^{+}$found $=308.09$, $\mathrm{C}_{16} \mathrm{H}_{12} \mathrm{~N}_{4} \mathrm{OS}$ requires 308.07 .

\section{3-(5-((1H-benzo[d]imidazol-2-ylthio)methyl)-1,3,4- oxadiazol-2-yl)aniline $5 \mathrm{~b}$ :}

Prepared from $m$-amino benzoic acid $(1.37 \mathrm{~g}, 0.01$ mol) as creamy coloured powder; yield: $46 \%$; IR, $\mathrm{cm}^{-1}$ : 3178.80 (NH str), 3085.45 (ArCH str), 1627.15 (C=N str), 1382.18 (NH bend), 1267.05 (COC str), 744.57 (CS str); ${ }^{1} \mathrm{H}$ NMR, $\delta 10.52$ (s, 1H, 1'H), 7.63 (d, 2H, $J=8.6$ $\mathrm{Hz}, 5$ ', 8'H), 7.38-7.42 (d, 2H, J=5.98 Hz, 2", 3’H), 7.21 (d, J=8.6 Hz, 2H, 6', 7'H), 6.86 (s, 1H, 2'H), 6.52 (m, 1H, 4"H), 3.8 (s, 2H, $\left.\mathrm{NH}_{2}\right), 4.32\left(\mathrm{~s}, 2 \mathrm{H}, \mathrm{SCH}_{2}\right)$; ${ }^{13} \mathrm{C}$ NMR, $\delta 163.2$ (C5) 156.3 (C2), 150.0 (C2'), 148.6 (C3”), 140.3 (C4', 9'), 130.5 (C5'), 129.3 (C1”), 124.2 (C6',7'), 118.7 (C4”), 117.3(C6”), 114.8 (C5', 8'), 113.5 (C2"), 34.7 (CS); MS, m/z: 323.09 $\left(100 \%, \mathrm{M}^{+}\right), 324.09\left(16.8 \%, \mathrm{M}^{+}+1\right), 325.08(5.2 \%$, $\left.\mathrm{M}^{+}+2\right),(\mathrm{M})^{+}$found $=323.09, \mathrm{C}_{16} \mathrm{H}_{13} \mathrm{~N}_{5} \mathrm{OS}$ requires 323.08 .

\section{4-(5-((1H-benzo[d]imidazol-2-ylthio)methyl)-1,3,4- oxadiazol-2-yl)aniline 5c:}

Prepared from $p$-amino benzoic acid $(1.37 \mathrm{~g}, 0.01$ mol) as brown crystals; yield: $41 \%$; IR, $\mathrm{cm}^{-1}: 3166.24$ (NH str), 3085.45 (ArCH str), 1598.89 (C=N str), 1374.38 (NH bend), 1262.42 (COC str), 742.04 (CS str); ${ }^{1} \mathrm{H}$ NMR, $\delta 12.52$ (s. 1H, 1'H), 7.58-7.53 (m, 4H, 5', 8', 2", 6’'H), 7.25 (d, 2H, J=8.4 Hz, 6', 7'H), 6.82 (d, J=6.42 Hz, 2H, 3", 5"H), 4.22 (s, 2H, $\mathrm{SCH}_{2}$ ), $3.68\left(\mathrm{~s}, 2 \mathrm{H}, \mathrm{NH}_{2}\right) ;{ }^{13} \mathrm{C} \mathrm{NMR}, \delta 164.2(\mathrm{C} 5), 159.6(\mathrm{C} 2)$, 148.3 (C2'), 144.9 (C4”), 140.1(C4', 9'), 128.2 (C2", 6"), 123.4 (C6', 7'), 115.8 (C1'), 114.7 (C5', 8', 3”, 5"), 35.3 (CS); MS, m/z: $323.07\left(100 \%, \mathrm{M}^{+}\right), 324.09$ $\left(17.3 \%, \mathrm{M}^{+}+1\right), 325.08\left(5.2 \%, \mathrm{M}^{+}+2\right),(\mathrm{M})^{+}$found $=$ 323.07, $\mathrm{C}_{16} \mathrm{H}_{13} \mathrm{~N}_{5} \mathrm{OS}$ requires 323.08.

\section{2-((1H-benzo [d]imidazol-2-ylthio) methyl)- 5-(2-chlorophenyl)-1,3,4-oxadiazole 5d:}

Prepared from $o$-chloro benzoic acid $(1.56 \mathrm{~g}, 0.01$ mol) as white powder; yield: $29 \%$; IR, $\mathrm{cm}^{-1}$ : 3156.68 ( NH str), 3085.45 (ArCH str), 1629.81 $(\mathrm{C}=\mathrm{N}$ str), 1344.88 ( $\mathrm{C}=\mathrm{N}$ bend), 740.60 (CS str), 637.01 (CCl str); ${ }^{1} \mathrm{H}$ NMR, $\delta 11.23$ (s, 1H, 1'H), 7.78 (d, $J=6.18 \mathrm{~Hz}, 1 \mathrm{H}, 6$ ' $\mathrm{H}$ ), 7.62-7.83 (m, 3H, 5', 8', 2'” $\mathrm{H}), 7.35-7.20(\mathrm{~m}, 4 \mathrm{H}, 6$ ', 7', 4', 5', $\mathrm{H}), 4.16$ $\left(\mathrm{s}, 2 \mathrm{H}, \mathrm{SCH}_{2}\right) ;{ }^{13} \mathrm{C} \mathrm{NMR}, \delta 163.6(\mathrm{C} 5), 157.8(\mathrm{C} 2)$, 150.1 (C2'), 137.5 (C4', 9'), 138.2 (C1”), 132.2 (C2"), 130.7 (C4”), 129.8 (C3”), 128.5 (C6"), 126.9 (C5”), 123.8 (C6', 7'), 115.3 (C5', 8'), 35.5 (CS); MS, m/z: $342.04\left(100.0 \%, \mathrm{M}^{+}\right), 343.02\left(16.9 \%, \mathrm{M}^{+}+1\right), 344.03$ (35.8\%, $\left.\mathrm{M}^{+}+2\right), 345.04\left(\mathrm{M}^{+}+3,7.5 \%\right), 346.03\left(\mathrm{M}^{+}+4\right.$, $1.9 \%)(\mathrm{M})^{+}$found $=342.04, \mathrm{C}_{16} \mathrm{H}_{11} \mathrm{ClN}_{4} \mathrm{OS}$ requires 342.03 .

\section{2-((1H-benzo[d]imidazol-2-ylthio)methyl)-5-(3-} nitrophenyl)-1,3,4-oxadiazole 5e:

Prepared from $m$-nitro benzoic acid $(1.67 \mathrm{~g}, 0.01$ mol) as brown powder; yield: 63\%; IR, $\mathrm{cm}^{-1}$ : 3136.63 ( $\mathrm{NH}$ str), 3085.45 (ArCH str), 1623.05 ( $\mathrm{C}=\mathrm{N}$ str), 1345.29 ( $\mathrm{NH}$ bend), 1264.32 (COC str), 896.33 (CS str), 740.60 ( $\mathrm{N}=\mathrm{O}$ sym str); ${ }^{1} \mathrm{H}$ NMR, $\delta$ 12.41 (s, 1H, 1'H), 8.43 (s, 1H, 2"H), 8.40 (t, 1H, 6"H), 8.31 (d, J=9.26 Hz, 1H, 4’H), 7.82 (t, 1H, 5”H), 7.57 (m, 2H, 5', 8'H), 7.18 (d, 2H, J=8.2 Hz, 6', 7'H), 4.40 $\left(\mathrm{s}, 2 \mathrm{H}, \mathrm{SCH}_{2}\right) ;{ }^{13} \mathrm{C} \mathrm{NMR}, \delta 165.2(\mathrm{C} 5), 159.3(\mathrm{C} 2)$, 150.0 (C2”), 148.3 (C3”), 138.9 (C4',9'), 132.8 (C6”), 131.5 (C5”), 124.0 (C1”), 123.1 (C2”,4”, 6', 7'), 115.9 (C5', 8'), 33.8 (CS); MS, m/z: $353.08\left(100.0 \%, \mathrm{M}^{+}\right)$, $354.07\left(23.1 \%, \mathrm{M}^{+}+1\right), 355.06\left(3.9 \%, \mathrm{M}^{+}+2\right),(\mathrm{M})^{+}$ found $=353.08, \mathrm{C}_{16} \mathrm{H}_{11} \mathrm{~N}_{5} \mathrm{O}_{3} \mathrm{~S}$ requires 353.06.

2-((1 H-benzo[d]imidazol-2-ylthio )methyl)-5(3-bromophenyl)-1,3,4-oxadiazole 5f:

Prepared from $m$-bromo benzoic acid $(2.01 \mathrm{~g}, 0.01 \mathrm{~mol})$ as brown powder; yield: $34 \%$; IR, $\mathrm{cm}^{-1}: 3180.63$ (NH str), 3085.45 (ArCH str), 1623.87 (C=N str), 1345.29 ( NH bend), 1265.88 (COC str), 738.14 (CS str), 516.53 (CBr str); ${ }^{1} \mathrm{H}$ NMR, $\delta 11.32$ (s, $1 \mathrm{H}, 1$ 'H), $7.98(\mathrm{~d}, 1 \mathrm{H}, J=6.78 \mathrm{~Hz}, 6$ "H), 7.62-7.41 (m, 5H, 5', 8', 2', 4', 5', $\mathrm{H}), 7.18$ (d, 2H, J=8.4 Hz, 6', 7'H), $4.17\left(\mathrm{~s}, 2 \mathrm{H}, \mathrm{SCH}_{2}\right) ;{ }^{13} \mathrm{C} \mathrm{NMR}, \delta 164.4$ (C5), 158.7 (C2), 150.2 (C2'), 139.0 (C4', 9'), 133.7 (C2'), 131.6 (C5”), 128.5 (C1", 4”), 126.2 (C5”), 122.1 (C6', 7'), 119.6 (C3”), 115.8 (C5', 8'), 34.9 (CS); MS, $m / z$ : $387.95\left(100.0 \%, \mathrm{M}^{+}\right), 388.97\left(16.3 \%, \mathrm{M}^{+}+1\right), 389.98$ (5.2\%, $\left.\mathrm{M}^{+}+2\right), 385.96$ (96.3\%), 386.99 (18.3\%), (M) found $=387.95, \mathrm{C}_{16} \mathrm{H}_{11} \mathrm{BrN}_{4} \mathrm{OS}$ requires 385.98 .

\section{4-(5-((1H-benzo[d]imidazol-2-ylthio)methyl)-1,3,4- oxadiazol-2-yl)phenol 5g:}

Prepared from $p$-hydroxy benzoic acid (1.38 g, $0.01 \mathrm{~mol})$ as pale yellow powder; yield: $25 \%$; IR, $\mathrm{cm}^{-1}: 3760.38$ (OH str), 3353.65 (NH str), 3085.45 (ArCH str), $1633.22(\mathrm{C}=\mathrm{N}$ stretch), 1386.04 (NH bend), 1252.97 (COC str), 739.95 (CS str); ${ }^{1} \mathrm{H}$ NMR, $\delta 10.53$ (s, 1H, 1'H), 8.27 (s, 1H, OH), 7.99 (d, 2H, J=7.27 Hz, 2", 6"H), 
$7.73\left(\mathrm{~d}, 2 \mathrm{H}, J=8.6 \mathrm{~Hz}, 5^{\prime}, 8{ }^{\prime} \mathrm{H}\right), 7.42(\mathrm{~d}, J=8.6 \mathrm{~Hz}$, 2H, 6', 7'H), 7.02 (d, 2H, J=7.27 Hz, 3', 5'”H), 3.55 (s, 2H, $\left.\mathrm{SCH}_{2}\right) ;{ }^{13} \mathrm{C} \mathrm{NMR}, \delta 164.5$ (C5), 162.3 (C2), 159.3 (C4”), 150.2 (C2'), 139.6 (C4', 9'), 123.9 (C6', 7'), 119.2 (C1"), 116.7 (C2", 3", 5”, 6”), 115.3 (C5', 8'), 36.2 (CS); MS, m/z: $324.05\left(100.0 \%, \mathrm{M}^{+}\right)$, $325.06\left(18.9 \%, \mathrm{M}^{+}+1\right), 326.07\left(6.3 \%, \mathrm{M}^{+}+2\right),(\mathrm{M})^{+}$ found $=324.05, \mathrm{C}_{16} \mathrm{H}_{12} \mathrm{~N}_{4} \mathrm{O}_{2} \mathrm{~S}$ requires 324.07.

\section{(E)-2-((1H-benzo[d]imidazol-2-ylthio)methyl)-5- styryl-1,3,4-oxadiazole 5h:}

Prepared from cinnamic acid $(1.48 \mathrm{~g}, 0.01 \mathrm{~mol})$ as yellowish powder; yield: $60 \%$; IR, $\mathrm{cm}^{-1}: 3353.65$ (NH str), 3085.45 (ArCH str), $1633.22(\mathrm{C}=\mathrm{N}$ str), 1386.04 (NH bend), 1252.97 (COC str), 739.95 (CS str); ${ }^{1} \mathrm{H}$ NMR, $\delta 11.58\left(\mathrm{~s}, 1 \mathrm{H}, 1{ }^{\prime} \mathrm{H}\right), 7.87-7.85$ (m, 4H, 5', 8', 2", 6'” $)$, 7.53-7.32 (m, 5H, 6', 7', 3", 4", 5"H), 7.28 (dd, J=14.35 Hz, 2H, olefinicH), $3.86\left(\mathrm{~s}, 2 \mathrm{H}, \mathrm{SCH}_{2}\right) ;{ }^{13} \mathrm{C} \mathrm{NMR}, \delta 163.2$ (C5), 158.6 (C2), 151.6 (C2'), 140.3 (C4', 9'), 137.8 (C1'), 134.1 (C olefinic), 129.2 (C2", 3", 5", 6"), 128.0 (C4"), 125.6 (C olefinic), 123.2 (C6',7'), 115.6 (C5',8'), 35.4 (CS); MS, $m / z$ : $334.10\left(100.0 \%, \mathrm{M}^{+}\right), 335.09$ (21.3\%, $\left.\mathrm{M}^{+}+1\right), 336.07\left(5.6 \%, \mathrm{M}^{+}+2\right),(\mathrm{M})^{+}$found $=334.10$, $\mathrm{C}_{18} \mathrm{H}_{14} \mathrm{~N}_{4} \mathrm{OS}$ requires 334.09.

\section{2-(5-((1H-benzo[d]imidazol-2-ylthio) methyl)- 1,3,4-oxadiazol-2-yl)phenol 5i:}

Prepared from salicylic acid $(1.38 \mathrm{~g}, 0.01 \mathrm{~mol})$ as black powder; yield: 47\%; IR, $\mathrm{cm}^{-1}: 3509.25$ (OH str), 3200.26 ( NH str), 3085.45 (ArCH str), 1603.07 (C=N str), 1345.49 (NH bend), 1244.69 (COC str), 740.10 (CS str); ${ }^{1} \mathrm{H}$ NMR, $\delta 10.52$ (s, 1H, 1'H), 8.02 (s, 1H, $\mathrm{OH})$, 7.69-7.62 (m, 3H, 5', 8', 6"'H), 7.28-7.0 (m, 5H, 6', 7', 3", 4', 5'H), 3.51 (s, 2H, $\left.\mathrm{SCH}_{2}\right) ;{ }^{13} \mathrm{C} \mathrm{NMR}, \delta$ 166.1 (C5), 161.8 (C2), 158.2 (C2”), 150.6 (C2'), 139.8 (C4', 9'), 130.5 (C4”), 127.8 (C6”), 123.3 (C6', 7'), 122.2 (C5"), 118.6 (C3"), 115.7 (C5',8'), 109.0 (C1"), 33.8 (CS); MS, $m / z: 324.05\left(100.0 \%, \mathrm{M}^{+}\right)$, $325.06\left(20.2 \%, \mathrm{M}^{+}+1\right), 326.04\left(3.0 \%, \mathrm{M}^{+}+2\right),(\mathrm{M})^{+}$ found $=324.05, \mathrm{C}_{16} \mathrm{H}_{12} \mathrm{~N}_{4} \mathrm{O}_{2} \mathrm{~S}$ requires 324.07.

3-(5-((1 H-benzo[d]imidazol-2-ylthio)methyl)1,3,4-oxadiazol-2-yl)-1-cyclopropyl-6-fluoro-7(piperazin-1-yl)quinolin-4(1H)-one 5j:

Prepared from ciprofloxacin $(3.31 \mathrm{~g}, 0.01 \mathrm{~mol})$ as yellowish powder; yield: $61 \%$; IR, $\mathrm{cm}^{-1}: 3200.26(\mathrm{NH}$ str), 3085.45 (ArCH str), 2973 (AliCH str), 1616.68 $(\mathrm{C}=\mathrm{N}$ str), 1363.81 ( $\mathrm{NH}$ bend), 1260.65 (COC str), 1178.33 (CF str), 741.62 (CS str); ${ }^{1} \mathrm{H}$ NMR, $\delta 11.23$ (s, 1H, 1'H), 8.67 (s, 1H, FCH), 7.85 (d, $J=8.8 \mathrm{~Hz}$, $\left.2 \mathrm{H}, 5^{\prime}, 8^{\prime} \mathrm{H}\right), 7.52$ (s, 1H, NCH, 1,4-dihydropyridine), 7.23 (d, $J=8.8 \mathrm{~Hz}, 2 \mathrm{H}, 6$ ', 7'H), 6.22 (s, 1H, FC$\mathrm{CH})$, 4.24-4.18 (m, 3H, cyclopropyl and $\left.\mathrm{SCH}_{2}\right)$, $3.48\left(\mathrm{t}, 4 \mathrm{H}, \mathrm{N}\left(\mathrm{CH}_{2}\right)_{2}\right.$ piperazinyl), 2.81 (t, $4 \mathrm{H}$, $\mathrm{N}\left(\mathrm{CH}_{2}\right)_{2}$ piperazinyl), 2.54 (s, 1H, piperazinyl), 1.381.18 (m, 4H, cyclopropyl); ${ }^{13} \mathrm{C}$ NMR, $\delta 176.3$ (CO, 1,4-dihydropyridine), 167.4 (C5), 159.0 (C2), 154.2 (CF), 150.1 (C2'), 148.3 (C-N=Ar), 142.2 (=C-N 1,4-dihydropyridine), 139.6 (C4', 9'), 135.0 (=C-N condensed $\mathrm{C}$ of 1,4-dihydropyridine), 124.3 (C6', 7'), 118.1 (C condensed Cof 1,4-dihydropyridine), 116.3 (=C 1,4-dihydropyridine), 113.2 (C5', 8'), 110.8 $(\mathrm{FC}=\mathrm{C}), 103.8(\mathrm{C}$ Ar), $49.5(\mathrm{~N}=\mathrm{C}$ piperazinyl), 46.3 (C-NH piperazinyl), 36.1 (CS), 33.9 (NC cyclopropyl), 8.1 (C cyclopropyl); MS, $m / z: 515.17\left(100.0 \%, \mathrm{M}^{+}\right)$, $516.16\left(31.7 \%, \mathrm{M}^{+}+1\right), 517.18\left(10.4 \%, \mathrm{M}^{+}+2\right), 518.18$ $(1.8 \%),(\mathrm{M})^{+}$found $=515.17, \mathrm{C}_{26} \mathrm{H}_{24} \mathrm{FN}_{7} \mathrm{O}_{2} \mathrm{~S}$ requires 515.19 .

\section{Preparation of pyridazine and phthalazine} derivatives $5 \mathrm{k}-\mathrm{m}$ :

A mixture of compound $4(2.67 \mathrm{~g}, 0.012 \mathrm{~mol})$, succinic anhydride $(1.0 \mathrm{~g}, 0.01 \mathrm{~mol})$ for compound $5 \mathrm{k}$, phthalic anhydride $(1.48 \mathrm{~g}, 0.01 \mathrm{~mol})$ for 51 and tetrachlorophthalic anhydride $(2.85 \mathrm{~g}, 0.01 \mathrm{~mol})$ for $5 \mathrm{~m}$ in absolute ethanol $(5 \mathrm{ml})$ and glacial acetic acid $(0.28$ $\mathrm{ml}, 0.005 \mathrm{~mol}$ ) was refluxed for $3 \mathrm{~h}$ and cooled. The reaction mixture was poured into ice water mixture and neutralized with dilute $\mathrm{NaHCO}_{3}$. The solid obtained was filtered and recrystallized from ethanol (95\%).

1 -(2-(1H-benzo[d]imidazol-2-ylthio)acetyl) pyridazine-3,6-dione $5 \mathrm{k}$ :

Pink powder; yield: 27\%; IR, $\mathrm{cm}^{-1}$ : 3349.19 (NH str), 3085.45 (ArCH str), 3072.56 (AliCH str), 2986 (AliCH str), 1831.94 (C=O str), 1638.19 ( $\mathrm{C}=\mathrm{N}$ str), 1329.65 ( $\mathrm{NH}$ bend), 731.71 (CS str); ${ }^{1} \mathrm{H}$ NMR, $\delta 12.54(\mathrm{~s}, 1 \mathrm{H}, 1$ 'H), $10.41(\mathrm{~s}, 1 \mathrm{H}, 2 \mathrm{H}), 8.02$ (d, $\left.J=8.4 \mathrm{~Hz}, 2 \mathrm{H}, 5^{\prime}, 8^{\prime} \mathrm{H}\right), 7.62$ (d, $J=8.4 \mathrm{~Hz}, 2 \mathrm{H}, 6$ ', 7'H), 4.36 (s, 2H, SCH2), $2.55(\mathrm{~s}, 4 \mathrm{H}, 4,5 \mathrm{H})$; ${ }^{13} \mathrm{C}$ NMR, $\delta 175.2$ (C3), 173.4 (C6), $168.8(\mathrm{C}=\mathrm{O})$, 148.1 (C2'), 139.5 (C4', 9'), 124.0 (C6', 7'), 115.3 (C5', 8'), 38.6 (CS), 29.1 (C4), 26.2 (C5); MS, m/z: $304.03\left(100.0 \%, \mathrm{M}^{+}\right), 305.04\left(15.9 \%, \mathrm{M}^{+}+1\right), 306.05$ $\left(6.2 \%, \mathrm{M}^{+}+2\right),(\mathrm{M})^{+}$found $=304.03, \mathrm{C}_{13} \mathrm{H}_{12} \mathrm{~N}_{4} \mathrm{O}_{3} \mathrm{~S}$ requires 304.06 .

2-(2-(1H-benzo[d]imidazol-2-ylthio)acetyl)-2,3dihydrophthalazine-1,4-dione 5l:

Black powder; yield: 35\%; IR, $\mathrm{cm}^{-1}: 3383.12$ (NH str), 
3166.96 (ArCH str), 1687.55 ( $\mathrm{C}=\mathrm{O}$ str), 1655.62 $\left(\mathrm{C}=\mathrm{N}\right.$ str), 1370.07 ( $\mathrm{NH}$ bend), $739.90\left(\mathrm{CS}\right.$ str); ${ }^{1} \mathrm{H}$ NMR, $\delta 11.62$ (s, 1H, 1'H), 7.86 (s, 1H, 2H), 7.60-7.56 (m, 4H, 5, 6, 7, 8H), 7.51 (d, J=8.6 Hz, 2H, 5', 8'H), 7.33 (d, J=8.6 Hz, 2H, 6', 7'H), 4.28 ( s, 2H, $\left.\mathrm{SCH}_{2}\right)$; ${ }^{13} \mathrm{C}$ NMR, $\delta 169.3$ (C=O), 168.1 (C3), 159.2 (C10), 147.8 (C2'), 140.1 (C4', 9'), 132.5 (C6, 7), 129.2 (C4, 9), 124.2 (C5, 8), 123.5 (C6', 7'), 115.9 (C5', 8'), 38.9 (CS); MS, m/z: $352.11\left(100.0 \%, \mathrm{M}^{+}\right), 353.10\left(21.3 \%, \mathrm{M}^{+}+1\right), 354.08$ $\left(6.3 \%, \mathrm{M}^{+}+2\right),(\mathrm{M})^{+}$found $=352.11, \mathrm{C}_{17} \mathrm{H}_{12} \mathrm{~N}_{4} \mathrm{O}_{3} \mathrm{~S}$ requires 352.06 .

\section{2-(2-(1H-benzo[d]imidazol-2-ylthio)acetyl)-5,6,7,8- tetrachloro-2,3-dihydrophthalazine-1,4-dione 5m:}

Yellowish white powder; yield: $39 \%$; IR, $\mathrm{cm}^{-1}: 3410.36$ ( $\mathrm{NH}$ str), 3053.17 (ArCH str), 1700.26 (C=O str), 1329.92 (NH bend), 739.85 (CS str), 648.26 (CCl str); ${ }^{1} \mathrm{H}$ NMR, $\delta 11.58$ (s, 1H, 1'-H), 8.63 (s, 1H, 2-H), 7.64 (d, $J=8.62 \mathrm{~Hz}, 2 \mathrm{H}, 5$ ', 8'-H), 7.36 (d, $J=8.62 \mathrm{~Hz}, 2 \mathrm{H}$, 6', 7'-H), 4.17 (s, 2H, $\left.\mathrm{SCH}_{2}\right) ;{ }^{13} \mathrm{C}$ NMR, $\delta 168.7$ $(\mathrm{C}=\mathrm{O}), 167.9$ (C3), 159.3 (C10), 147.8 (C2'), 139.4 (C4', 9'), 135.8 (C6, 7), $133.6(\mathrm{C} 4,9), 128.9$ (C5, 8), 124.0 (C6', 7'), 115.7 (C5', 8'), 38.3 (CS); MS, m/z: $487.93\left(75.8 \%, \mathrm{M}^{+}\right), 488.91\left(13.9 \%, \mathrm{M}^{+}+1\right), 489.9$ (100\%, M $\left.{ }^{+}+2\right), 490.9$ (20.6\%), 491.8 (52.8\%), 492.9 (10.0\%), $493.8(12.7 \%), 494.9$ (2.7\%), $(\mathrm{M})^{+}$found = 487.93, $\mathrm{C}_{17} \mathrm{H}_{8} \mathrm{Cl}_{4} \mathrm{~N}_{4} \mathrm{O}_{3} \mathrm{~S}$ requires 487.91 .

\section{Antimicrobial activity:}

In the initial tube containing $380 \mu \mathrm{l}$ of BHI broth, $20 \mu \mathrm{l}$ of compound (representing $100 \mu \mathrm{g}$ of compound) from the stock solution prepared by dissolving $10 \mathrm{mg}$ of test compound in $2 \mathrm{ml} \mathrm{DMSO}$, was added. For dilutions, $200 \mu \mathrm{l}$ of BHI broth was added into the next 9 tubes separately. Then from the initial tube, $200 \mu 1$ was transferred to the second tube containing $200 \mu 1$ of BHI broth. This was considered as $10^{-1}$ dilution. From $10^{-1}$ diluted tube (second tube) $200 \mu 1$ was transferred to third tube to make $10^{-2}$ dilutions. The serial dilution was repeated up to $10^{-9}$ dilutions for each compound. In each serially diluted tube, $200 \mu 1$ of microbial culture suspension in BHI $\left(10^{5}\right.$ bacteria/fungi per $\left.\mathrm{ml}\right)$ was added. Ciprofloxacin/fluconazole and control tests with solvent DMSO were performed under similar condition for comparison. The tubes were incubated at $37^{\circ}$ for antibacterial and $25^{\circ}$ for antifungal activities for $24 \mathrm{~h}$ and observed for turbidity. The MIC was defined as lowest concentration of compound, which prevented the formation of turbidity.

\section{Antitubercular activity:}

First, $200 \mu \mathrm{l}$ of sterile deionised water was added to all outer perimeter wells of sterile 96 wells plate to minimize the evaporation of medium in the test wells during incubation. The wells received $100 \mu \mathrm{l}$ of the Middlebrook 7H9 broth and serial dilution of compounds dissolved in DMSO were made directly on plate from 100 to $0.2 \mu \mathrm{g} / \mathrm{ml}$. The above said wells were inoculated with $100 \mu \mathrm{l}$ of $2000 \mathrm{cfu} / \mathrm{ml}$ of organisms in Middlebrook $7 \mathrm{H} 9$ broth. Plates were covered and sealed with Parafilm and incubated at $37^{\circ}$ for $5 \mathrm{~d}$. After this, $25 \mu \mathrm{l}$ of freshly prepared 1:1 mixture of AlamarBlue reagent and $10 \%$ Tween 80 was added to the plate and incubated for $24 \mathrm{~h}$. The same method was followed for control DMSO and INH. A blue colour in the well was interpreted as no bacterial growth, and pink colour was scored as growth. The MIC was defined as lowest drug concentration, which prevented the colour change from blue to pink.

\section{RESULTS AND DISCUSSION}

The synthetic routes leading to title compounds $5 \mathrm{a}-\mathrm{j}$ and $5 \mathrm{k}-\mathrm{m}$ were depicted in figs. 1 and 2 . Well versed with the intrinsic antimicrobial property of sulphur, $1 \mathrm{H}-2-$ mercapto benzimidazole 1 was aptly used as the starting material to incorporate 1H-benzimidazol-2-ylthio methyl motif into the target molecules. 2-(1-H-benzo[d] imidazol-2ylthio) acetic acid 2 was obtained through William's reaction of 1 with chloroacetic acid in the presence of sodium hydroxide ${ }^{[8,25]}$. On treatment with thionyl chloride, 2 yielded the acid chloride, 2-(1-H-benzo[d]imidazol-2ylthio)acetyl chloride $3^{[26,27]}$. 2-(1-H-benzo[d]imidazol-2-ylthio)aceto hydrazide 4 was prepared by reacting 3 with hydrazine hydrate $^{[28]}$. The 4 was further cyclodehydrated with different substituted aromatic acids in presence of phosphorous oxychloride as dehydrating agent to generate 2-(benzimidazol-2ylthiomethyl)-5-aryl1,3,4-oxadizoles 5a-j in moderate to good yields. The other dehydrating agents used for this purpose include sulphuric acid, phosphoric acid, trifluoroacetic acid, phosphorus pentachloride, phosphorus pentoxide, thionyl chloride and milder reagents such as carbodiimide derivatives, $\mathrm{TsCl} /$ pyridine, trimethylsilyl chloride, $\mathrm{Ph}_{3} \mathrm{O} / \mathrm{Tf}_{2} \mathrm{O}, \mathrm{PPh}_{3} / \mathrm{CX}_{4}(\mathrm{X}=\mathrm{Cl}, \mathrm{Br}, \mathrm{I})$ and Burgess reagent ${ }^{[2,30]}$. The mechanism of formation of compounds $5 \mathrm{a}-\mathrm{j}^{[30]}$ is outlined in fig. 3. In another route, 4 on condensation with succinic, phthalic and tetrachlorophthalic anhydrides in presence of catalytic amount of acetic acid resulted in the formation of corresponding pyridazine or phthalazine diones ${ }^{[31]}$ 

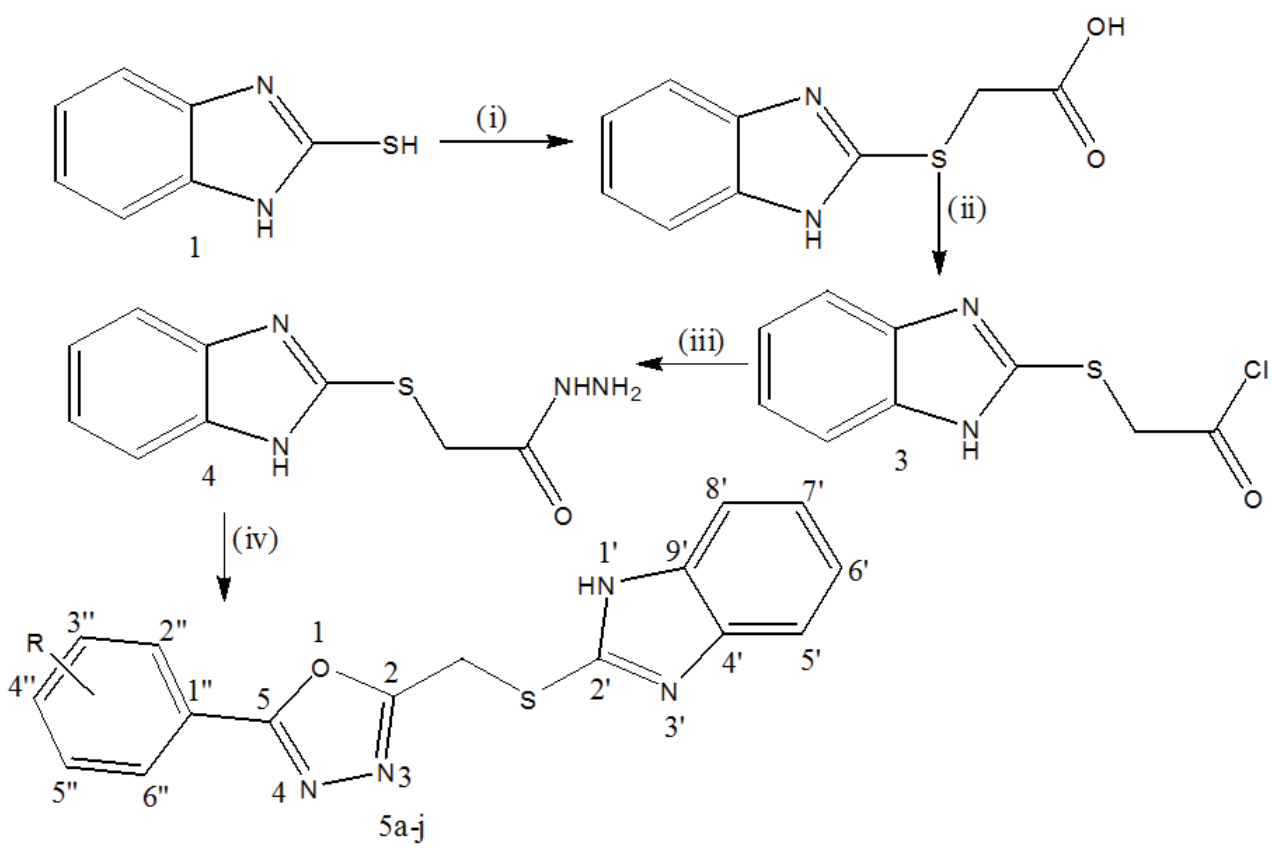

Fig. 1: Synthetic route for compounds $5 \mathrm{a}-\mathbf{j}$

Reagents: i. $\mathrm{ClCH}_{2} \mathrm{COOH} / \mathrm{NaOH}$; ii. $\mathrm{SOCl}_{2}$; iii. $\mathrm{NH}_{2} \mathrm{NH}_{2} \mathrm{H}_{2} \mathrm{O}$; iv. $\mathrm{ArCOOH} / \mathrm{POCl}_{3}$

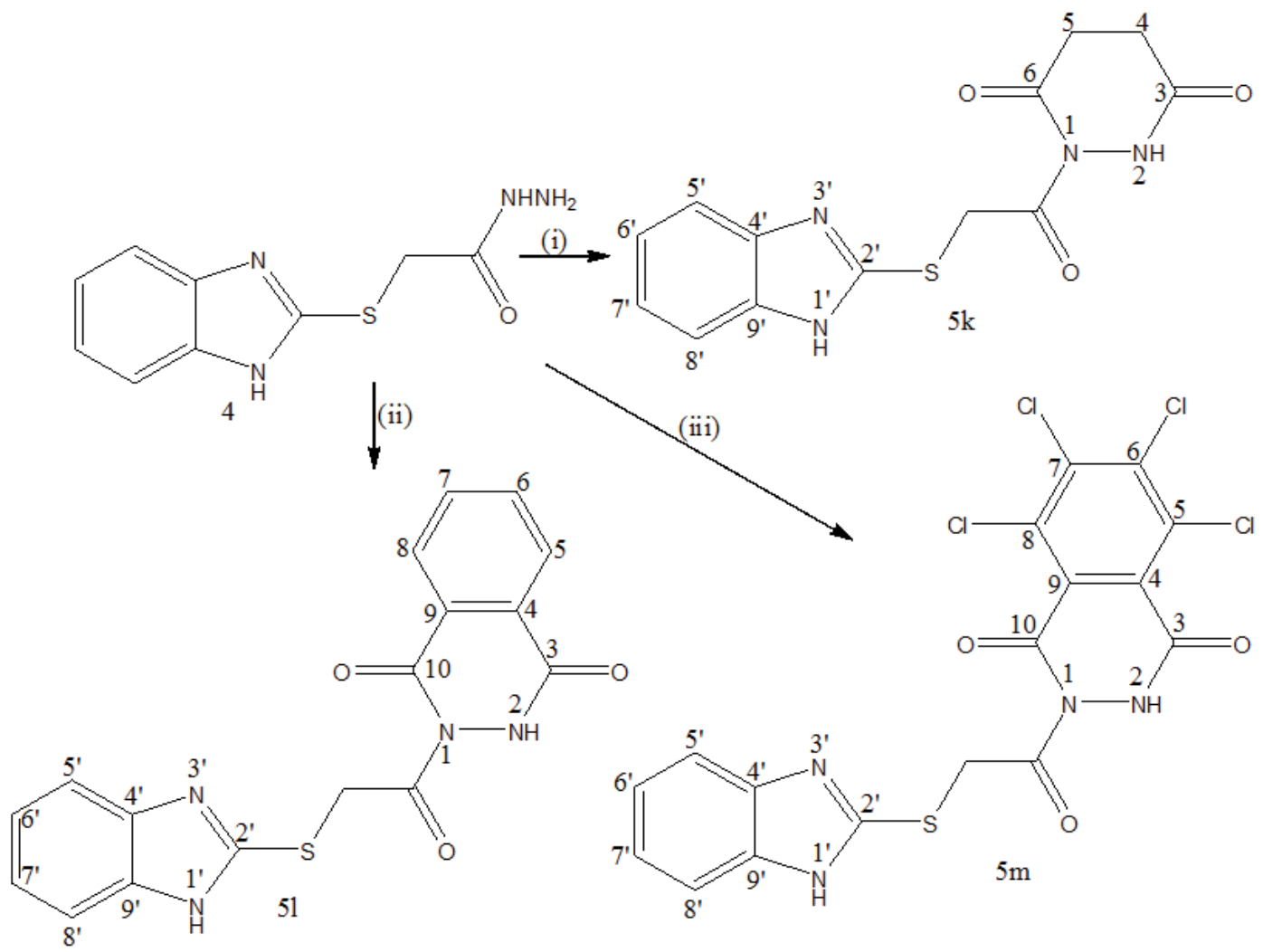

Fig. 2: Synthetic route for compounds $5 \mathrm{k}-\mathrm{m}$

Reagents: i. succinic anhydride/AcOH; ii. phthalic anhydride/AcOH; iii. tetrachl orophthalic anhydride/AcOH

viz.,1-(2-(1H-benzo[d]imidazol-2-ylthio)acetyl) pyridazine/(tetrachloro)phthalazine diones $5 \mathrm{k}, 51$ and $5 \mathrm{~m}$, respectively in moderate yields. The synthesized compounds were purified by recrystallization from ethanol as more or less amorphous powders. The $\mathrm{R}_{\mathrm{f}}$ values and melting points of the compounds 5a-m are presented in Table 1.

The structure of the synthesized compounds was determined on the basis of their IR, NMR and mass spectral data. The IR spectrum of 3 exhibited the 


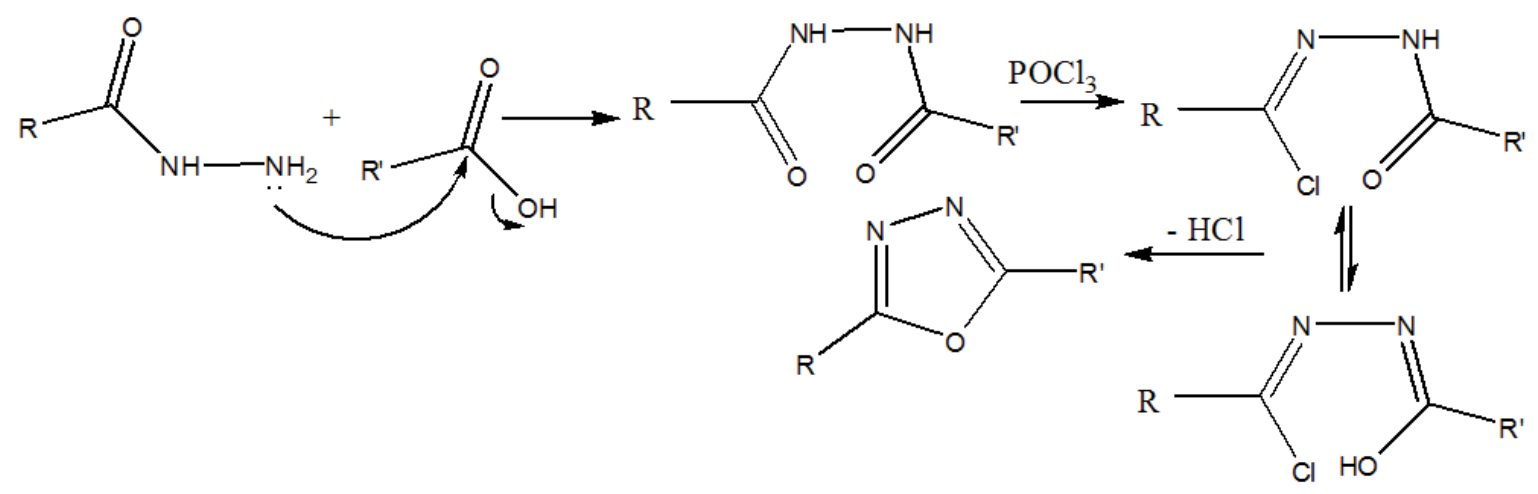

Fig. 3: Mechanism of formation of 2,5-disubstituted-1,3,5-oxadiazoles

TABLE 1: PHYSICOCHEMICAL DATA OF COMPOUNDS (5 a-m)

\begin{tabular}{|c|c|c|c|}
\hline Entry & $\mathrm{Ar}$ & $R_{f}$ value & $\mathrm{MP}^{\circ}$ \\
\hline $5 a$ & & 0.70 (Pet. ether:benzene 7:3) & $190-192$ \\
\hline $5 b$ & & 0.56 (Pet. ether:benzene 8:2) & $272-274$ \\
\hline $5 c$ & & 0.53 (Pet. ether:benzene 7:3) & $226-228$ \\
\hline $5 d$ & & 0.72 (Pet. ether:benzene 2:8) & $282-284$ \\
\hline $5 e$ & & 0.91 (Pet. ether:benzene 3:7) & 167-169 \\
\hline $5 f$ & & 0.55 (Pet. ether:benzene 1:9) & $171-173$ \\
\hline $5 g$ & & 0.36 (Pet. ether:benzene 3:7) & $211-213$ \\
\hline $5 \mathrm{~h}$ & & 0.62 (Pet. ether:benzene 8:2) & $232-234$ \\
\hline $5 i$ & & 0.35 (Pet. ether:benzene 3:7) & $278-279$ \\
\hline $5 j$ & & 0.45 (Pet. ether:benzene 3:7) & $213-214$ \\
\hline $5 k$ & -- & 0.63 (Pet. ether:benzene 3:7) & $295-296$ \\
\hline $5 l$ & . & 0.65 (Pet. ether:benzene 3:7) & $234-236$ \\
\hline $5 \mathrm{~m}$ & -- & 0.52 (Pet. ether:benzene 3:7) & $284-285$ \\
\hline
\end{tabular}


bands at $1792 \mathrm{~cm}^{-1}$ due to $\mathrm{C}=\mathrm{O}$ stretch, $682 \mathrm{~cm}^{-1}$ ascribing to $\mathrm{C}-\mathrm{Cl}$ stretch, $769 \mathrm{~cm}^{-1}$ corresponding to $\mathrm{C}-\mathrm{S}$ stretch, $3145 \mathrm{~cm}^{-1}$ pertaining to aromatic $\mathrm{C}-\mathrm{H}$ stretch and $3452 \mathrm{~cm}^{-1}$ attributing to $\mathrm{N}-\mathrm{H}$ stretch. The compounds 5a-j showed bands in IR spectra at 3136$3383 \mathrm{~cm}^{-1}$ characteristic to $\mathrm{NH}$ stretch, 3053-3166 $\mathrm{cm}^{-1}$ because of aromatic C-H stretch, $1598-1655 \mathrm{~cm}^{-1}$ due to $\mathrm{C}=\mathrm{N}$ stretch, $1252-1265 \mathrm{~cm}^{-1}$ corresponding to C-O-C asymmetric stretch and 731-744 $\mathrm{cm}^{-1}$ due to C-S stretch. In their IR spectra, 5k-m displayed bands at $3349-3410 \mathrm{~cm}^{-1}$ pertaining to N-H stretch, $1657-1687 \mathrm{~cm}^{-1}$ ascribing to $\mathrm{C}=\mathrm{O}$ stretch, $1638-1655 \mathrm{~cm}^{-1}$ corresponding to $\mathrm{C}=\mathrm{N}$ stretch, $1329-1370 \mathrm{~cm}^{-1}$ attributing to $\mathrm{N}-\mathrm{H}$ deformation and 731-739 $\mathrm{cm}^{-1}$ due to C-S stretch. The ${ }^{1} \mathrm{H}$ NMR spectra of $5 \mathrm{a}-\mathrm{m}$ exhibited a broad singlet at $\delta$ 12.71-10.41 representing benzimidazole $\mathrm{NH}$, aromatic hydrogens resonated in the range of $\delta 8.5-6.10$, a singlet at $\delta$ 4.6-3.9 corresponding to two hydrogens of $\mathrm{SCH}_{2}$ and at $\delta \sim 8.0$ representing pyridazine/phthalazine $\mathrm{NH}$ amongst other protons. The ${ }^{13} \mathrm{C}$ NMR spectra of compounds exhibited peaks due to aromatic carbons at $\delta \sim 160-110$, carbonyl carbons at $\delta 176-165$, carbon of $\mathrm{SCH}_{2}$ at $\delta \sim 40$ and aliphatic carbon atoms at $\delta \sim 50-8$. The $\mathrm{M}^{+}$and $/$or $\mathrm{M}^{+}+1$ and $\mathrm{M}^{+}+2$ peaks showed by $5 \mathrm{a}-\mathrm{m}$ in mass spectra were in close agreement with their molecular weights.

The title compounds 5a-m were evaluated for antimicrobial activity against $S$. aureus, E. faecalis (Gram-positive bacteria), K. pneumonia, E. coli (Gramnegative bacteria) and C. albicans and A. niger (fungi) by serial dilution method ${ }^{[32]}$ on BHI broth medium. The antimicrobial screening results presented in Table 2 were mean of triplicate reading. Only 5a showed MIC $6.25 \mu \mathrm{g} / \mathrm{ml}$, indicating better activity than standard drug ciprofloxacin whereas, $5 \mathrm{j}$ with $12.5 \mu \mathrm{g} / \mathrm{ml}$, 5e and $5 \mathrm{f}$ with $25 \mu \mathrm{g} / \mathrm{ml}$ displayed moderate activity against $S$. aureus. Electron withdrawing substituents such as nitro and bromo sustained the activity. All the compounds showed potent activity against E. faecalis, exhibiting $0.2 \mu \mathrm{g} / \mathrm{ml} \mathrm{MIC}$ in comparison to $10 \mu \mathrm{g} / \mathrm{ml}$ for ciprofloxacin. It seems, substitutions on phenyl ring at the 2-position of 1,3,4-oxadiazole has no effect on activity against $E$. faecalis. All compounds tested had very poor activity against $E$. coli exhibiting no dependency on substituents except one compound. Only $5 \mathrm{j}$ showed $0.2 \mu \mathrm{g} / \mathrm{ml}$ MIC against $K$. pneumoniae, indicating a greater potency than ciprofloxacin (MIC $10 \mu \mathrm{g} / \mathrm{ml}$ ). Since $5 \mathrm{j}$ contains ciprofloxacin fragment in it, the activity seems to be better than ciprofloxacin itself. Eventually, 5j emerged out to possess good activity against both Gram-positive and Gram-negative bacteria. In general, the title compounds displayed good activity against Gram-positive organisms relative to against the Gram negative. This could perhaps be due to poor cell wall penetration of the compounds.

Against C. albicans, 5g, 51 and 5m exhibited good antifungal activity (MIC $50 \mu \mathrm{g} / \mathrm{ml}$ ) than rest of the compounds. However, they were less active than standard antifungal fluconazole (MIC $30 \mu \mathrm{g} / \mathrm{ml}$ ). Phthalazine derivatives were found to be better acting than their pyridazine counterpart. All the compounds displayed an outstanding antifungal activity with MIC

TABLE 2: ANTIMICROBIAL ACTIVITY OF COMPOUNDS (5 a-m)

\begin{tabular}{|c|c|c|c|c|c|c|c|c|}
\hline \multirow{2}{*}{ Compound } & \multirow{2}{*}{ CLogP } & \multicolumn{7}{|c|}{ MIC, $\mu \mathrm{g} / \mathrm{ml}$} \\
\hline & & S. aureus & E. faecalis & E. coli & K. pneumoniae & A. niger & C. albicans & M. tuberculosis \\
\hline $5 a$ & 2.82 & 6.25 & 0.2 & 100 & 100 & 0.2 & 100 & 12.8 \\
\hline $5 b$ & 1.86 & 50 & 0.2 & 100 & 100 & 0.2 & 100 & 25.6 \\
\hline $5 c$ & 1.50 & 50 & 0.2 & 100 & 100 & 0.2 & 100 & 51.2 \\
\hline $5 d$ & 3.31 & 50 & 0.2 & 100 & 100 & 0.2 & 100 & 51.2 \\
\hline $5 e$ & 2.63 & 25 & 0.2 & 100 & 100 & 0.2 & 100 & 51.2 \\
\hline $5 f$ & 3.71 & 25 & 0.2 & 100 & 100 & 0.2 & 100 & 51.2 \\
\hline $5 g$ & 2.44 & 50 & 0.2 & 100 & 100 & 0.2 & 50 & $>100$ \\
\hline $5 \mathrm{~h}$ & 3.41 & 50 & 0.2 & 100 & 100 & 0.2 & 100 & 51.2 \\
\hline $5 i$ & 2.14 & 100 & 0.2 & 100 & 100 & 0.2 & 100 & 12.8 \\
\hline $5 j$ & 1.14 & 12.5 & 0.2 & 100 & 0.2 & 0.2 & 100 & 25.6 \\
\hline $5 k$ & 0.79 & 100 & 0.2 & 100 & 100 & 0.2 & 100 & 51.2 \\
\hline $5 l$ & 3.88 & 100 & 0.2 & 100 & 100 & 0.2 & 50 & 51.2 \\
\hline $5 m$ & 6.37 & 100 & 0.2 & 100 & 100 & 0.2 & 50 & 51.2 \\
\hline Ciprofloxacin & -0.72 & 10 & 10 & 10 & 10 &.-- &.-- &.-- \\
\hline Fluconazole & 0.52 & --- & --- & --- & --- & 30 & 30 & --- \\
\hline $\mathrm{INH}$ & -0.67 & --- & -- & --- & --- & --- & --- & 0.4 \\
\hline
\end{tabular}


$0.2 \mu \mathrm{g} / \mathrm{ml}$ against $A$. niger than fluconazole. Here also, the activity is found to be independent of substituents. Among the title compounds, $5 \mathrm{j}$ showed selectivity towards bacteria whereas; phthalazine 51 and $5 \mathrm{~m}$ displayed somewhat selectivity towards fungi.

The in vitro antitubercular activity of compounds was assessed against $M$. tuberculosis $H 37 R v$ by microplate alamar blue assay ${ }^{[33]}$. The activity data is shown in Table 2. The compounds showed very weak antitubercular activity. Only $5 \mathrm{a}$ and $5 \mathrm{i}$ showed MIC $12.8 \mu \mathrm{g} / \mathrm{ml}$ vis-à-vis $0.4 \mu \mathrm{g} / \mathrm{ml}$ for standard drug INH. The poor antitubercular activity by title compounds could probably be due to their lower lipophilicity, indicated by their $\mathrm{C} \log \mathrm{P}$ values, and thereby reduced cell wall permeation. Thus, among the title compounds, 5a with phenyl substitution and 5 i with 2-hydroxy phenyl substitution emerged to be moderately acting against all the tested bacteria, fungi and mycobacterium.

In conclusion, a new series of 1H-benzimidazol-2ylthiomethyl moiety incorporated hybrid molecules containing 2-substitutituted 1,3,4-oxadiazole, pyridazin-3,6-dione and phthalazin-1,4-dione were synthesized successfully by a facile protocol via acid hydrazide intermediate and were spectrally characterized. The target compounds showed excellent to moderate to poor preliminary in vitro antimicrobial and very feeble in vitro antitubercular activity against tested bacterial and fungal strains. The results showcased the significance of molecular hybridisation leading to bioactive hybrid molecules. However, synthesis of many more derivatives in the series and their activity testing would usher new trends in structural optimization of these compounds. Further, quantitative structure-activity relationship (QSAR) studies would probably give a better insight into biochemical mode of action and pattern of activity of the synthesized compounds. Also, the template considered in the study is found to have anticancer effect $^{[34]}$, the title compounds would also be explored as anticancer agents in addition to antimicrobial molecules warranting a powerful incentive for further research.

\section{Acknowledgements:}

The authors thank SAIF, Punjab University, Chandigarh for providing NMR and Manipal College of Pharmaceutical Sciences, Manipal for mass spectra.
They are grateful to Prof. KG Bhat for expertise in antimicrobial activity.

\section{Conflict of interest:}

The authors declare no conflict of interest with respect to this work.

\section{Financial support and sponsorship:}

Nil.

\section{REFERENCES}

1. Petri WA Jr. Penicillins, cephalosporins, and other $\beta$-lactam antibiotics. In: Brunton LL, Chabner BA, Knollman BC, editors. Goodman and Gilman's The Pharmacological basis of Therapeutics. 12th ed. New York: McGraw-Hill; 2011. p. 1491-93.

2. Valverde MG, Torroba T. Sulfur-nitrogen heterocycles. Molecules 2005;10:318-20.

3. Kus C, Sozudonmez F, Altanlar N. Synthesis and antimicrobial activity of some novel 2-[4-(substituted piperazin-/piperidin1-ylcarbonyl)phenyl]-1H-benzimidazole derivatives. Arch Pharm Chem Life Sci 2009;342:54-60.

4. Sondhi SM, Singh N, Kumar A, Lozach O, Meijer L. Synthesis, antiinflammatory, analgesic and kinase (CDK-1, CDK-5 and GSK-3) inhibition activity evaluation of benzimidazole/ benzoxazole derivatives and some Schiff's bases. Bioorg Med Chem 2006;14:3758-65.

5. Kus C, Atlanlar N. Synthesis of some new benzimidazole carbamate derivatives for evaluation of antifungal activity. Turk J Chem 2003;27:35-9.

6. Desai NC, Shihori NR, Kotadiya GM, Desai P. Synthesis, antibacterial and antitubercular activities of benzimidazole bearing substituted 2-pyridone motifs. Eur J Med Chem 2014;82:480-9.

7. Kim JS, Sun Q, Gatto B. Structure-activity relationships of benzimidazoles and related heterocycles as topoisomerase inhibitors. Bioorg Med Chem 1996;6:4621-30.

8. Shingalapur RV, Hosamani KM, Keri RS, Hugar MH. Derivatives of benzimidazole pharmacopore: synthesis, anticonvulsant, antidiabetic and DNA cleavage studies. Eur J Med Chem 2010;45:1753-9.

9. Mavrova AT, Anichina KK, Vucher DI, Tsenov JA, Denkova PS, Kondeva MS, et al. Antihelminthic activity of some newly synthesized 5(6)-(un)substituted-1H-benzimidazol2-ylthioacetylpiperazine derivatives. Eur J Med Chem 2006;41:1412-20.

10. Ram S, Wise DS, Townsend LB. Synthesis of 2-thiobenzimidazole derivatives as potential antifilarial agents. J Heterocycl Chem 1985;22:1269-74.

11. Joshi N, Korgaokar S, Parekh H. Synthesis and antimicrobial activity of substituted 1,3,4-oxadiazoles. Indian J Hetrocycl Chem 1996;5:241-2.

12. Shaikh A, Meshram J. Novel 1,3,4-oxadiazole derivatives of dihydropyrimidinones: Synthesis, antiinflammatory, anthelmintic and antibacterial activity evaluation. J Heterocycl Chem 2016;53:1176-82.

13. Bakht MA, Islam M, Siddiqui AA. Synthesis and antifungal activity of some new 1,3,4-oxadiazoles. Indian J Heterocycl Chem 2006;15:297-8. 
14. Kazemizadeh, AR, Shajari N, Shapouri R, Adibpour N, Teimuri-Mofrad R. Synthesis and antibrucella activity of some new 1,3,4-oxadiazole derivatives containing a ferrocene unit. J Iran Chem Soc 2016;13:1349-55.

15. Sotelo E, Fraiz N, Yanez M, Laguna R, Cano E, Jose B, et al. Pyridazines Part 28: 5-alkylidene-6-phenyl-3(2H)pyridazinones, a new family of platelet aggregation inhibitors. Bioorg Med Chem 2002;12:1575-7.

16. Sung KJ, Lee HJ, Suh ME, Choo HY, Lee SK, Park HJ, et al. Synthesis and cytotoxicity of 1-substituted-2-methyl-1Himidazo[4,5-g]phthalazine-4,9-dione derivatives. Bioorg Med Chem 2004;12:3683-6.

17. Wu J, Song B, Chen H, Bhadury P, Hu D. Synthesis and antifungal activity of 5-chloro-6-phenyl-pyridazine-3(2H)one derivatives. Molecules 2009;14:3676-87.

18. Hansch C, Leo A. QSAR in metabolism. In: Heller SR, editor. Exploring QSAR Fundamentals and applications in chemistry and biology. Washington DC: American Chemical Society; 1995.

19. Hansch C, Gao H. Comparative QSAR: Radical reactions of benzene derivatives in chemistry and biology. Chem Rev 1997;97:2995-3060.

20. Nandi S, Bagchi M. Importance of Kier-Hall topological indices in the QSAR of anticancer drug design. Curr Comp Aided Drug Des 2012;8:159-70.

21. Viegas-Jr C, Danuello A, da Silva Bolzani V, Barreiro EJ, Fraga CAM. Molecular hybridization: a useful tool in the design of new drug prototypes. Curr Med Chem 2007;14:1829-52.

22. Fraga CAM. Drug hybridization strategies: before or after lead identification? Expert Opin Drug Disc 2009;4:605-9.

23. Bosquesi PL, Melo TRF, Vizioli EO, dos Santos JL, Chung MC. Antiinflammatory drug design using a molecular hybridization approach. Pharmaceuticals 2011;4:1450-74.

24. http://media.cambridgesoft.com/support/14/ ChemDrawActiveXEnterpriseConstantNA140.msi

25. Bespalov AY, Gorchakova TL, Ivanov AY, Kuznetsov MA,
Kuznetsova LM, Pankova AS. et al. Alkylation and aminomethylation of 1,3-dihydro- $2 \mathrm{H}$-benzimidazole-2thione Chem Heterocycl Comp 2015;50:1547-58.

26. March J. Advanced organic chemistry reactions, mechanisms and structure. 4th ed. New Delhi: Wiley India Pvt Ltd; 2005. p. 437-8.

27. Murugan V, Prasad KR, Sarma GVSR, Ramanathan M, Suresh B. Synthesis of triazole, thiadiazole and oxadiazole bearing 2-thiomethyl benzimidazole and their biological evaluation. Indian J Heterocycl Chem 2001;11:169-70.

28. Elrayess RA, Ghareb N, Azab MM, Said MM. Synthesis and antimicrobial activities of some novel benzimidazole and benzotriazole derivatives containing $\beta$-lactam moiety. Life Sci J 2013;10:1784-93.

29. de Oliveira CS, Lira BF, Barbosa-Filho JM, Lorenzo JGF, de Athayde-Filho PF. Synthetic approaches and pharmacological activity of 1,3,4-oxadiazoles: a review of the literature from 2000-2012. Molecules 2012;17:10192-231.

30. Padmavathi V, Reddy GS, Mohan AVN, Mahesh K. Synthesis of symmetrical and unsymmetrical 1,3,4-oxadiazoles and their inter conversion to 1,3,4-thiadiazoles and 1,2,4-triazoles. Arkivoc 2008;2008:48-60.

31. Hedaya E, Hinman RL, Theodoropulos S. Preparation and properties of some new N, N'- biisoimides and their cyclic isomers: reaction of N, N'-biisomaleimide with dienes. J Org Chem 1966;31:1317-26.

32. Schwalve R, Moore LS, Goodwin AC. Antimicrobial Susceptibility Testing Protocols. Oxford: CRC Press; 2007.

33. Collins EA, Franzblow SG. Microplate alamar blue assay versus BACTEC 460 system for highthroughput screening of compounds against Mycobacterium tuberculosis and Mycobacterium avium. Antimicrob Agents Chemother 1997;41:1004-9.

34. Mochona B, Mazzio E, Gangapuram M, Mateeva N, Redda KK. Synthesis of some benzimidazole derivatives bearing 1,3,4-oxadiazole moiety as anticancer agents. Chem Sci Trans 2015;4:534-40. 